\title{
Ultra Long Construct Minimally Invasive Spinal Stabilization Using Percutaneous Pedicle Screws in the Treatment of Symptomatic Multicentric Spinal Metastasis
}

\author{
Chee Kean Lee, Chris Yin Wei Chan, Mun Keong Kwan \\ Department of Orthopaedic Surgery, Faculty of Medicine, University of Malaya, Kuala Lumpur, Malaysia
}

\begin{abstract}
Managing multiple level spinal metastases is challenging. We report the case of a 58-year-old female with advanced lung cancer who presented with multiple pathological fractures of the thoracic spine (T5, T6, T7, and T8 vertebrae). She was treated with palliative radiotherapy. Her resting pain improved, but the instability pain persisted. One month later, she had a trivial fall leading to a pathological fracture of the $\mathrm{L} 2$ vertebra with cauda equine syndrome. The patient was treated surgically with minimally invasive decompression of the L2 and with percutaneous instrumented stabilization using an ultra-long construct from T3 to L5 (15 spinal levels), spanning the previously radiated zone and the decompression site. Postoperatively, she had significant improvements in pain and neurology. There were no surgical complications. Ultra long construct minimally invasive spinal stabilization is the ideal approach for symptomatic multicentric spinal metastasis with poor prognostic scores. Using this technique, the goals of spinal stabilization and direct neural decompression can be achieved with minimal morbidity.
\end{abstract}

Keywords: Ultra long construct; Minimally invasive stabilization; Percutaneous; Spinal metastasis; Spinal cord compression

\section{Introduction}

Symptomatic multicentric spinal metastasis in patients with poor prognostic scores poses a dilemma for surgeons. We present the case of a metastatic lung carcinoma patient with severe instability pain due to pathological fractures over the T6, T7, and T8 vertebrae, which had been irradiated prior to the onset of cauda equina syndrome secondary to a L2 vertebra pathological fracture. The surgical risk for this patient would be overwhelming with conventional open surgery. With the introduction of percutaneous pedicle screws, we would like to introduce the concept of ultra long construct minimally invasive stabilisation (MISt) as a treatment option for this group of patients.

\section{Technical Note}

A 58-year-old lady with lung carcinoma presented with spinal metastasis involving the T5, T6, T7, T8, and L2 vertebrae, with pathological fracture of the T6, T7 and T8 vertebrae causing instability back pain without neurological deficits. The visual analogue scale (VAS) for her pain was 8 out of 10. She also had multiple liver metastases,

Received Jan 30, 2015; Revised Feb 24, 2015; Accepted Feb 28, 2015

Corresponding author: Chee Kean Lee

Department of Orthopaedic Surgery, Faculty of Medicine, University of Malaya,

50603, Lembah Pantai, Kuala Lumpur, Malaysia

Tel: +60379492061, Fax: +60379494642, E-mail: kenetto@hotmail.com 
but her liver function tests remained normal. Her Tomita score was 10 and her Tokuhashi score was 5 . The Spinal Instability Neoplastic Score (SINS) for the T5, T6, T7, T8, and L2 vertebrae were 6, 11, 14, 6 and 4, respectively. The option of percutaneous pedicle screw stabilisation was offered, but she opted for radiotherapy and received 5 fractions of radiotherapy to T5 to T8. Her instability pain persisted with VAS of 6/10 after the radiotherapy. She was still able to ambulate independently.

One month later, she had a fall and developed lower back pain in addition to weakness of the bilateral lower limbs (Frankel C) with urinary and bowel incontinence. Magnetic resonance imaging showed a new L2 fracture with cauda equina compression (Fig. 1). We performed an ultra long construct MISt with fluoroscopic guided percutaneous pedicle screws anchored proximally at T3, spanning the irradiated skin overlying T5 to T8, instrumentation at $\mathrm{T} 9$ and $\mathrm{T} 11$, a mini open direct decompres- sive surgery and vertebroplasty at L2 and distal anchorage at L3 and L5 (Fig. 2).

The operative time was 255 minutes with blood loss of $680 \mathrm{~mL}$. She was able to sit up on day 3, with improvement in her back pain. Her wounds healed well. Her neurology improved to Frankel D at 3 weeks and she achieved urinary continence at 6 weeks. Her pain score (VAS) improved further to $3 / 10$ and 2/10 at 2 weeks and 6 weeks postoperation, respectively. She passed away 7 months after her surgery due to progression of her lung lesion.

\section{Discussion}

Seventy percent of cancer patients have spinal metastases [1]. Ten percent of patients present with instability back pain with or without neurological deficits [2]. The surgical decision is governed by three factors: the life expectancy, the surgical indication, and the surgical risk. Tomita et al.

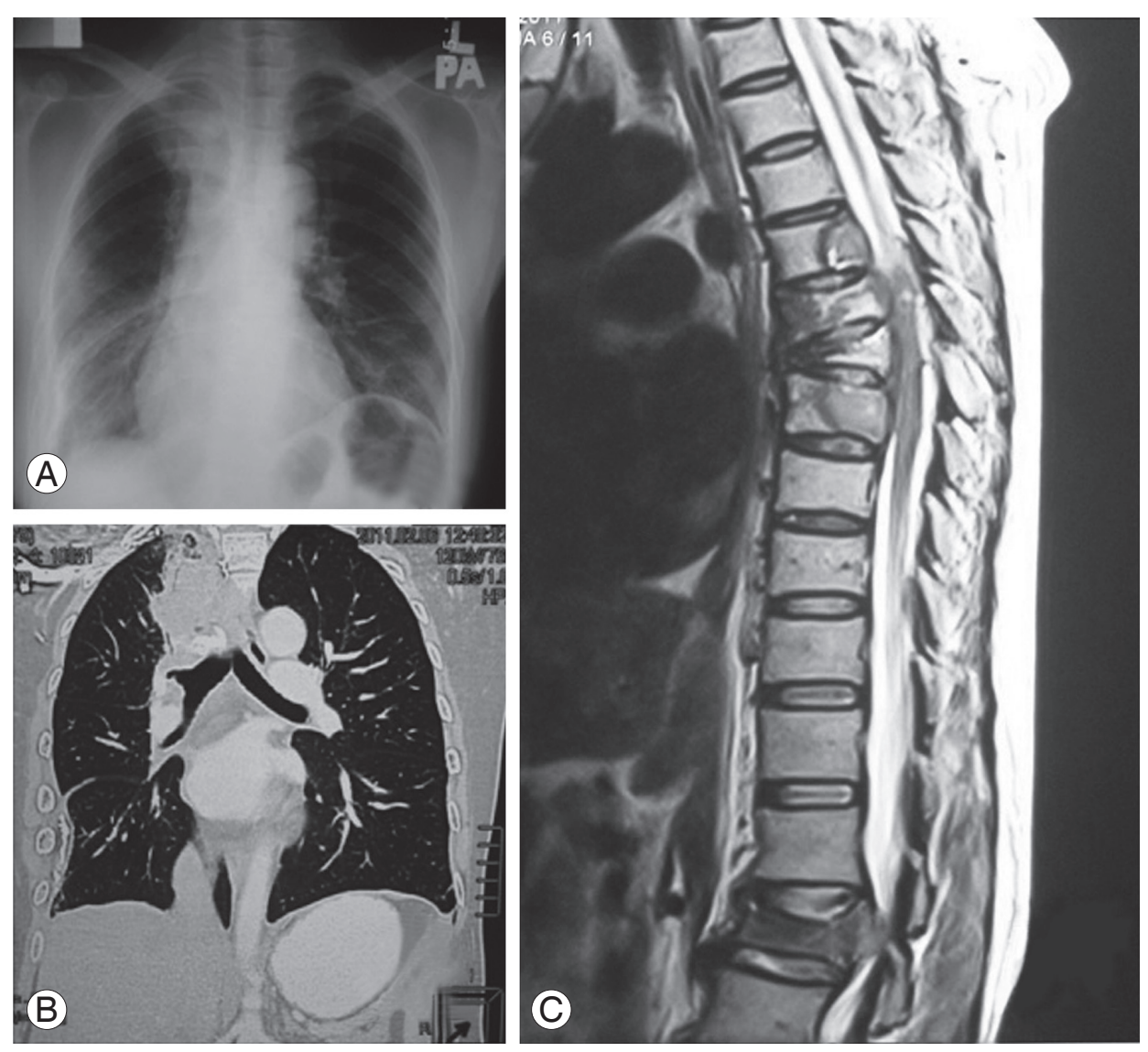

Fig. 1. (A, B) Chest radiograph and computed tomography scan showed an apical right lung adenocarcinoma. (C) Magnetic resonance imaging scan showed multiple spine metastases with multiple old fractures at T6, T7, and T8, which were radiated by the radio-oncologist. A new fracture at L2 vertebrae resulted in cauda equina syndrome. 

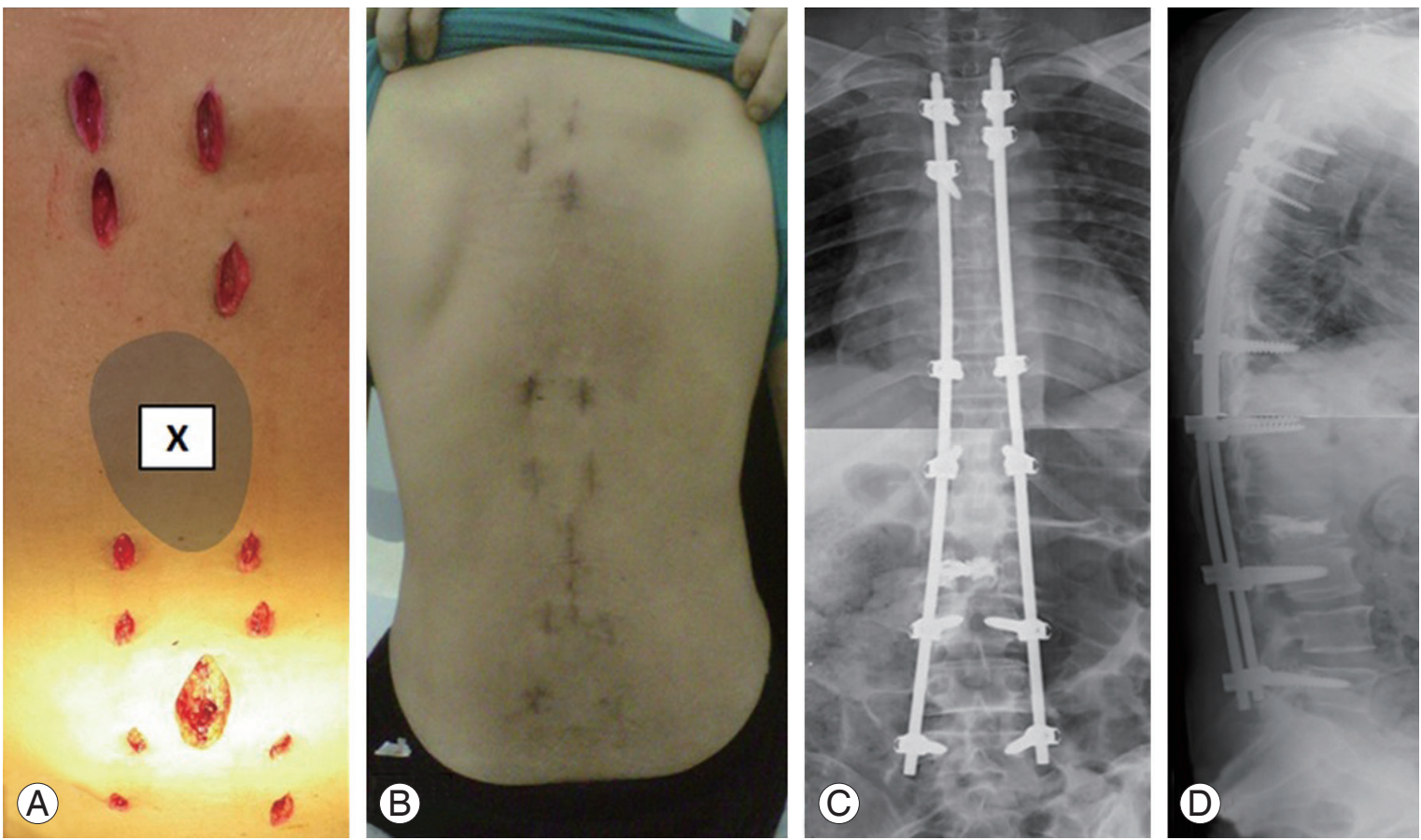

Fig. 2. (A) Intraoperative photo showed multiple small step wounds, indicating the insertion sites for the percutaneous pedicle screws. A minimally invasive decompression was performed for the fractured $L 2$ vertebrae. Notice that there was no incision over the radiated zone X. (B) Postoperative 2 weeks, the patient was able to sit up and ambulate with complete recovery from cauda equina syndrome (C, D) Postoperative radiograph showed minimally invasive stabilisation from T3-L5.

[3] and Tokuhashi et al. [4] recommended that patients with Tomita scores of 8 to 10 or Tokuhashi scores of 8 or less be treated with non-operative supportive care. However, Patchell at al. [5] and Lee et al. [6] demonstrated better outcomes for patients with metastatic epidural spinal cord compression (MESCC) who underwent direct surgical decompression with adjuvant radiotherapy compared to radiotherapy alone.

The clinical problem in this case was symptomatic multicentric spinal metastasis with spinal instability at T6, T7 and T8 and MESCC at L2. This clinical presentation typically mandates surgery. However, the survival prognostic scores were poor due to extensive disease. This, coupled with the morbidity of open surgery through the irradiated zone raised a dilemma. Lee et al. [6] reported a complication rate of $12.4 \%$ in patients undergoing surgery for MESCC. In patients who had previous radiotherapy, the risk of complications is even higher $[7,8]$.

Percutaneous pedicle screw systems are able to span long spinal segments in a minimally invasive manner. In this case, the construct spanned 15 spinal segments and we coined the term ultra long construct minimally invasive spinal stabilisation to describe the procedure. This system is ideal as we were able to avoid surgical incision over the radiotherapy site (Fig. 2), stabilize T6, T7, and T8, and perform direct decompressive surgery at L2. The operative intervention was completed with minimal morbidity to the patient.

However, ultra long construct MISt is challenging. This technique requires percutaneous pedicle screw insertion over the proximal thoracic vertebrae, which has the smallest pedicle dimensions [9]. Obtaining a view of the lateral projection of the vertebrae on the image intensifier is difficult in this region. This view is mandatory for the safety of percutaneous pedicle screws. Contouring and insertion of the rod percutaneously spanning a long segment also requires familiarity with minimally invasive instrumentation techniques. A minimally invasive approach for direct decompression would then complement this technique.

The MISt technique can be used to stabilize diseased spines, but does not allow for fusion of the diseased segment. The durability of the implants in patients with long survival is questionable. Thus, larger series with longer survival periods is necessary.

In conclusion, ultra long construct MISt is ideal for symptomatic multicentric spinal metastasis with poor 
prognostic scores. Using this technique, spinal stabilization and direct neural decompression can be achieved with minimal morbidity.

\section{Conflict of Interest}

No potential conflict of interest relevant to this article was reported.

\section{References}

1. Coleman RE. Clinical features of metastatic bone disease and risk of skeletal morbidity. Clin Cancer Res 2006;12:6243s-6249s.

2. Byrne TN. Spinal cord compression from epidural metastases. N Engl J Med 1992;327:614-9.

3. Tomita K, Kawahara N, Kobayashi T, Yoshida A, Murakami H, Akamaru T. Surgical strategy for spinal metastases. Spine (Phila Pa 1976) 2001;26:298-306.

4. Tokuhashi Y, Matsuzaki H, Toriyama S, Kawano H, Ohsaka S. Scoring system for the preoperative evaluation of metastatic spine tumor prognosis. Spine
(Phila Pa 1976) 1990;15:1110-3.

5. Patchell RA, Tibbs PA, Regine WF, et al. Direct decompressive surgical resection in the treatment of spinal cord compression caused by metastatic cancer: a randomised trial. Lancet 2005;366:643-8.

6. Lee CH, Kwon JW, Lee J, et al. Direct decompressive surgery followed by radiotherapy versus radiotherapy alone for metastatic epidural spinal cord compression: a meta-analysis. Spine (Phila Pa 1976) 2014;39:E587-92.

7. Wise JJ, Fischgrund JS, Herkowitz HN, Montgomery D, Kurz LT. Complication, survival rates, and risk factors of surgery for metastatic disease of the spine. Spine (Phila Pa 1976) 1999;24:1943-51.

8. Ghogawala Z, Mansfield FL, Borges LF. Spinal radiation before surgical decompression adversely affects outcomes of surgery for symptomatic metastatic spinal cord compression. Spine (Phila Pa 1976) 2001;26: 818-24.

9. Panjabi MM, Takata K, Goel V, et al. Thoracic human vertebrae. Quantitative three-dimensional anatomy. Spine (Phila Pa 1976) 1991;16:888-901. 